\title{
Desarrollo de un sistema aumentativo y adaptativo para apoyar el proceso de lectoescritura para alumnos con discapacidad visual de educación superior
}

Carmen Cerón, Etelvina Archundia, Beatriz Beltrán, Patricia Cervantes, José Galindo

\author{
Benemérita Universidad Autónoma de Puebla, \\ Facultad de Ciencias de la Computación, México \\ \{mceron, etelvina, bbeltran\} @cs.buap.mx, \\ \{cervantes.patty, jgalindo2016\} @gmail.com
}

\begin{abstract}
Resumen. El propósito de este artículo es presentar el diseño y desarrollo de un Sistema Adaptativo como herramienta de apoyo para el desarrollo de las Competencias de comunicación oral y escrita en los estudiantes con discapacidad visual. La metodología utilizada fue de Prototipos y el Diseño Centrado en el Usuario, se usaron Objetos de Aprendizaje e Interfaces Naturales de Usuario como el Kinect de Microsoft. Para el desarrollo se usaron las tecnologías de OpenNI de Kinect, MySQL, Greennhouse SDK, C++, C\# y se integró un conversor texto-voz (TTS) en Java. Finalmente se presentan los resultados obtenidos de una prueba piloto con un grupo focal de estudiantes con discapacidad visual bajo distintos escenarios de uso y de aprendizaje.
\end{abstract}

Palabras clave: Sistema accesible, aprendizaje adaptativo, objetos de aprendizaje, interfaces naturales de usuario, competencias.

\section{Development of an Augmentative and Adaptive System to Support the Process of Reading and Writing for Visually Disabled Students in Higher Education}

\begin{abstract}
The purpose of this article is to present the design and development of an Adaptive System like a help tool for the development the communicative oral and written Competence in the visually disabled students. The methodology used was the prototypes and the design centered in the user, it was used the learning objects and interfaces natural of the users like the Kinect of Microsoft. For the development was used the technologies of OpenNI of Kinect, MySQL, Greenhouse SDK, C++, C\# and was to integrate to converter text-voice (TTS) in Java. Finally, is present the results obtained in the pilot test with focal group technique of students with visual disability under different settings of use and learning.
\end{abstract}

Keywords. Accessible system, adaptive learning, learning objects, competency. 


\section{Introducción}

Actualmente las Tecnologías de la Información y Comunicación se consideran como herramientas de apoyo para las personas con alguna discapacidad en sus actividades diarias ya que les permiten acceder a diferentes ámbitos como son: educación, trabajo, comunicación y entretenimiento [1]. En México, la población en edad escolar que presenta problemas visuales es de un $20 \%$ y en términos mundiales los errores de refracción no corregidos (miopía, hipermetropía, astigmatismo y presbicia) constituyen la causa más importante de la discapacidad visual. De acuerdo con el Instituto Nacional de Estadística y Geografía (INEGI) la segunda discapacidad en el país es la visual, la primera es la motriz, existiendo otras discapacidades del hablar o comunicarse, escuchar, atender o aprender [2]. Por lo cual se requiere atender en las instituciones educativas a los estudiantes con alguna discapacidad visual para brindar mejores condiciones y herramientas para su vida académica universitaria. Por otra parte, lograr una mayor inclusión del uso de las Tecnologías en sus actividades para reducir la brecha digital y propiciar el desarrollo de distintas competencias genéricas y disciplinares. Una de las necesidades que se han identificado en la Educación Superior para una mayor inclusión de esta población es la de acondicionar espacios, aulas, desarrollar materiales didácticos, ayudas de software y recursos que puedan utilizarse en la modalidad presencial de forma accesible y permitan enriquecer el desarrollo de las competencias en el alumno en el proceso de enseñanza-aprendizaje.

Esta investigación tiene como propósito el diseño y desarrollo de un Sistema Aumentativo y Adaptativo Interactivo para el Proceso de Lectura y Escritura (SIS$A D A P-L E)$ como herramienta de apoyo para el desarrollo de las Competencias de Comunicación oral y escrita en los estudiantes con discapacidad visual por medio de Objetos de Aprendizaje Adaptativos e Interfaces Naturales de Usuario, integrando el uso del dispositivo del Kinect para facilitar la estimulación física y cognitiva por el reconocimiento postural del cuerpo humano, el uso gestual y reconocimiento de voz, adaptándose a las necesidades y limitaciones de los usuarios para lograr el acceso al sistema.

$\mathrm{Su}$ aportación permitirá apoyar el proceso aprendizaje y desarrollo de habilidades en la comunicación escrita y oral en los estudiantes universitarios con discapacidad visual para poder fortalecer sus competencias lectoras mediante actividades interactivas.

Para el diseño y desarrollo del Sistema Adaptativo se utilizó la metodología de Prototipos y el Diseño Centrado en el Usuario, el modelado en UML, las tecnologías de MySQL, Greennhouse SDK y se integró un conversor texto-voz (TTS) utilizando las herramientas de Java, $\mathrm{C}++\mathrm{y} \mathrm{CH}$.

El artículo está organizado como se indica a continuación:

En la sección 2 se presenta la fundamentación y la revisión teórica del trabajo de las Tecnologías de Apoyo para la Discapacidad Visual, Sistemas Adaptativos Hipermedia, la Tecnología de Interfaces Naturales de Usuario y del conversor de texto-voz.

En la sección 3 se define el análisis y el diseño del sistema en UML, la arquitectura del sistema, la implementación del sistema con las tecnologías OpenNI de Kinect, MySQL, Greennhouse SDK, C++ y C\# y Java para el reconocimiento de gestos y 
voz. En la sección 4 se muestran los resultados de la prueba piloto y las pruebas de funcionalidad y de valoración por los estudiantes con discapacidad del grupo focal desde un enfoque metodológico cualitativo. Finalmente se presentan las conclusiones y el trabajo a futuro de esta investigación.

\section{Estado del arte}

En esta sección se revisan los tópicos de Discapacidad Visual, Tecnologías de Apoyo, Sistemas Hipermedia Adaptativos, y las Interfaces Naturales de Usuario, los cuales sustentan a esta investigación.

\subsection{Discapacidad visual}

En el 2001, de acuerdo con la Clasificación Internacional del Funcionamiento, de la Discapacidad y de la Salud, presentada por la Organización Mundial de la Salud las personas con discapacidad "son aquellas que tienen una o más deficiencias físicas, mentales, intelectuales o sensoriales y que al interactuar con distintos ambientes del entorno social pueden impedir su participación plena y efectiva en igualdad de condiciones a las demás" [3]. La Discapacidad visual (DV) está relacionada con una deficiencia del sistema de la visión que afecta la agudeza visual, campo visual, motilidad ocular, visión de los colores o profundidad, afectando la capacidad de una persona para ver. Al hablar de DV podemos referirnos a la persona que presenta ceguera o baja visión. Algunos autores han definido términos como "dificultad visual severa", "deficiencia visual grave", "visión subnormal", "visión parcial", "visión residual", entre otros, para definir el espacio intermedio entre la visión normal y la ausencia total o casi total de visión, caracterizado por un sistema visual con alteraciones irreversibles y una pérdida en la capacidad visual que constituye un obstáculo para el desarrollo de la vida de las personas [3]. Por lo cual tenemos dos clasificaciones:

- Baja visión (hasta agudeza visual de 6/18). Es una condición de vida que disminuye la agudeza o el campo visual de la persona; es decir, que quienes presentan una baja visión ven significativamente menos que aquéllas que tienen una visión normal. Siendo esta una condición que no limita a quien la padece en su capacidad para desplazarse y conducirse de la forma que lo hace una persona con una visión optima, impidiendo que las personas que le rodean comprendan las dificultades que esta condición representa para realizar todas aquellas actividades que exigen una agudeza visual mayor, considerándolos apáticos, lentos, descuidados, incomodos o latosos. El docente debe estar abierto a la posibilidad de que dentro de su grupo haya estudiantes con Baja Visión, por lo que es recomendable que identifique a este tipo de personas para ofrecer apoyos.

- Ceguera (agudeza visual menor a 20/200). Es una condición de vida que afecta la percepción de imágenes en forma total reduciéndose en ocasiones a una mínima percepción de luz, impidiendo que la persona ciega reciba información visual del mundo que le rodea. 


\subsection{Tecnologías de apoyo y sistemas aumentativos y alternativos}

La Tecnología y de las Ciencias Computacionales en la última década se han inclinado por apoyar el área de las ciencias de la salud y la educación. La tendencia es aportar aplicaciones para apoyar a las personas con discapacidad y lograr mayor inclusión en su entorno. A partir de lo anterior ha surgido el término de Tecnología de Apoyo también conocida como Tecnología de Acceso o Rehabilitación.

Para Alcantud la define como "Todos aquellos aparatos, utensilios, herramientas, programas de ordenador o servicios de apoyo que tienen como objetivo incrementar las capacidades de las personas que, por cualquier circunstancia, no alcanzan los niveles medios de ejecución que por su edad y sexo le corresponderían en relación con la población normal" [4]. Por otra parte, la Norma UNE 139802:2003 le llamo "ayudas técnicas de software". Sin embargo, en 2007, dentro de ISO 9999:2007, estableció que ya no se tienen que llamar ayudas técnicas, sino "Tecnologías de Apoyo" [5], las cuales incluyeron: lectores de pantalla, magnificadores de pantalla, navegadores accesibles y navegadores alternativos.

Por otra parte de acuerdo a la ayuda que proporcionan y funcionalidad son conocidos como Sistemas Alternativos y Aumentativos de Acceso a la Información del Entorno: Son sistemas que engloban las ayudas o apoyos para personas con discapacidad visual y/o auditiva, modifican la señal, aumentándola o cambiando su modalidad para poder ser percibido por ellos.

Cuando existe un problema sensorial, la opción es el aumento de la señal que el contexto envía al sujeto. Los sistemas aumentativos se dirigen hacia la población con déficits visuales y auditivos mientras que los sistemas alternativos son medios que permiten, a quienes presentan la imposibilidad de alcanzar la información mediante una determinada modalidad sensorial, cambiar la naturaleza de la misma de modo que pueda aprehenderse mediante una modalidad que la persona mantiene funcional. $\mathrm{Al}$ gunas de estas son los medios tecnológicos para escritura en Braille, Tecnologías del Habla: El reconocimiento de voz y la conversión texto-voz. Actualmente se están incorporando otras tecnologías inalámbricas para la manipulación y el control del entorno como son las tabletas, iPad y las consolas (Wii, Play Station, Kinect) las cuales promueven una mayor interactividad y autonomía a las personas con discapacidad, las cuales están siendo aplicadas en la rehabilitación y para apoyar procesos de aprendizaje en la educación.

\subsection{Sistemas hipermedias adaptativos (SHA) y aprendizaje adaptativo}

El Aprendizaje Adaptativo recolecta información sobre los hábitos de aprendizaje, conocimientos, debilidades y fortalezas de cada usuario para crear un camino de aprendizaje a la medida de los alumnos teniendo en consideración sus dificultades y se adapta a la forma y ritmo de aprendizaje de cada uno, logrando tener un aprendizaje diferenciado y adaptativo único para cada estudiante.

Para Gaudioso define los SHA como "aquellos sistemas de hipermedia capaces de ajustar su presentación y navegación a las diferencias de los usuarios, reduciendo así los problemas de desorientación y falta de comprensión, propios de los sistemas hi- 
permedia no adaptativos" [6]. Para Brusilovsky "Un sistema se considera adaptativo cuando se adapta de forma automática y de forma personalizada a las necesidades del usuario [7]. Por lo que el SHA permite personalizar la información almacenada y la presenta a los usuarios según sus preferencias, conocimientos e intereses [8].

El SHA aplicado a la educación permite guiar a los estudiantes con discapacidad adaptando los contenidos y la navegación de acuerdo a las características y necesidades del usuario logrando incluir interfaces naturales de usuario para permitir una interacción adaptativa de acuerdo al dispositivo con el cual puede utilizar más el usuario teniendo en consideración su discapacidad, como en la Figura 1 se muestra.

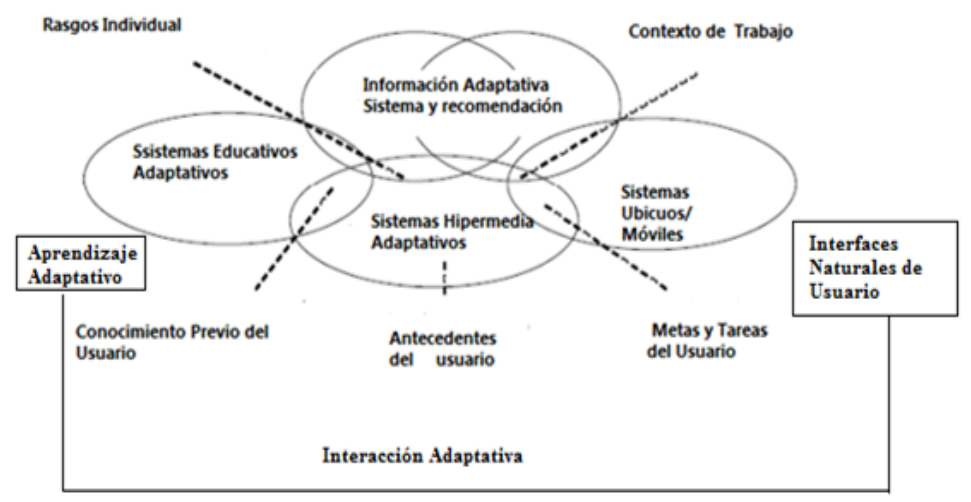

Fig. 1. Adaptación del sistema hipermedia de Brusilovsky con interfaces naturales.

\subsection{Interfaces naturales de usuario}

Las interfaces naturales de usuario (NUI) son aquellas en las que se interactúa con un sistema sin utilizar sistemas de mando o dispositivos de entrada de las GUI (Interfaces de Usuario Graficas) como sería el ratón, teclado alfanumérico, joystick, etc., y en su lugar, se hace uso de movimientos gestuales tales como las manos o el cuerpo que fungen como el mismo mando de control, algunos ejemplos son el reconocimiento a través de sensores de voz, de mirada, de movimiento y multitáctiles [9].

Existen varios dispositivos que son utilizados para implementar las NUI como son:

1. Microsoft Kinect Sensor, es un dispositivo que integra una gran cantidad de sensores que detectan el movimiento corporal del usuario que se coloca enfrente de él, al mismo tiempo captura el sonido permitiendo la interacción utilizando instrucciones de voz [10].

2. Leap Motion Controller: es una pequeña barra que se conecta a la computadora y detecta el movimiento de las manos para interactuar con la máquina [11].

3. Myo Gesture Control Armband: es una banda que se coloca en el antebrazo y por medio de los movimientos en el brazo realiza la interacción con la computadora [12]. 
4. Google Glass: son unos lentes creados por Google por medio de los cuales los usuarios solicitan y reciben información o servicios utilizando su voz, localización, video, entre otras variables que los lentes perciben [13].

Por lo que en esta investigación se enfoca al dispositivo del Kinect como medio de reconocimiento de gestos tanto de movimiento como de comando de voz. Según Wigdor y Wixon [14] consideran que las interfaces naturales de usuario se basan en crear interfaces que generen experiencias para poder usar la tecnología de acuerdo a las necesidades y contexto del usuario. Así mismo recomiendan ciertas pautas para el diseño de interfaces naturales tales como:

- Crear experiencias que den la sensación que es parte "de su cuerpo" como una extensión y lograr esa autonomía.

- Crear experiencias para todos usuarios desde nivel de principiante o experto de acuerdo a las características y perfil del usuario.

- Crear interfaces que considere contextos, metáforas, indicaciones visuales y propiciar escenarios con experiencias reales de su contexto.

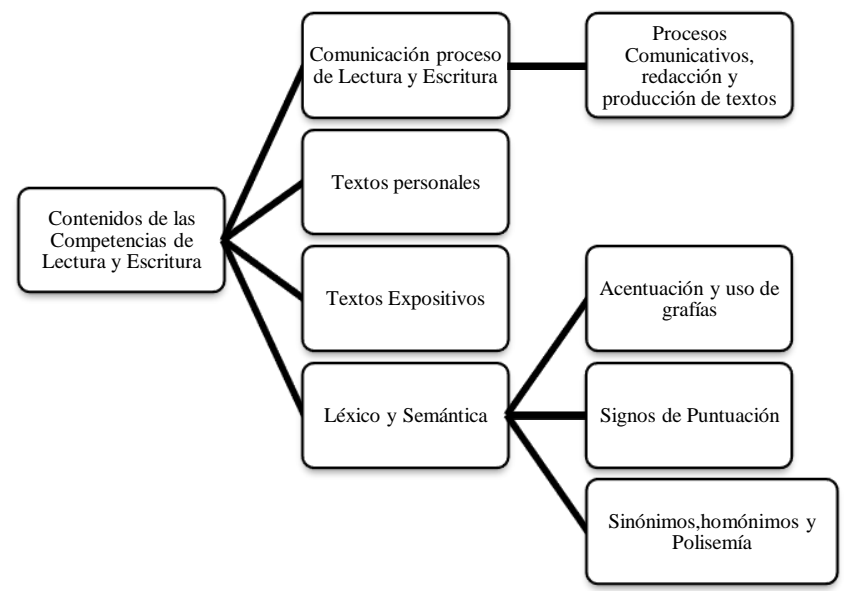

Fig. 2. Contenidos para el proceso de lectura y escritura del sistema.

\subsection{Competencias del proceso de lectura y escritura}

Para Tobón [15] define las competencias "como procesos complejos de desempeño integral con idoneidad en determinados contextos, que implican la articulación de diversos saberes, para realizar actividades y/o resolver problemas con sentido de reto, motivación, flexibilidad, creatividad y comprensión, dentro de una perspectiva de mejoramiento continuo y compromiso ético". Para este sistema las competencias de lectura y escritura a considerar y los contenidos a abordar en el sistema se muestran en la Figura 2. 


\section{Análisis y diseño del sistema aumentativo y adaptativo para apoyar el proceso de lectura y escritura (SIS-ADAP-LE)}

\subsection{Diseño centrado en el usuario}

Para el diseño del prototipo se utilizó el Diseño Centrado en el Usuario (DCU) que sitúa al estudiante con discapacidad visual en el núcleo del proceso de diseño de la interfaz. Para lo cual se realizó una serie de entrevistas y encuestas con el grupo focal, finalmente se plantearán los prototipos de la aplicación con balsamiq. Para el análisis y el diseño del sistema se determinaron los Casos de Uso del sistema en UML (ver Figura 3). El sistema permite identificar dos usuarios para el acceso y manipulación del sistema:

- Usuario Docente: Puede realizar consultas generales, alta de materiales y modificación con respecto a los contenidos, actividades y evaluación del tema.

- Usuario Estudiante con baja visión/Ceguera: Consulta los contenidos y materiales de información, al realizar el diagnóstico, el sistema adapta las interfaces y presenta los contenidos que requiere aprender y propone una serie de actividades lúdicas para el desarrollo de las competencias del proceso de lectura y escritura.

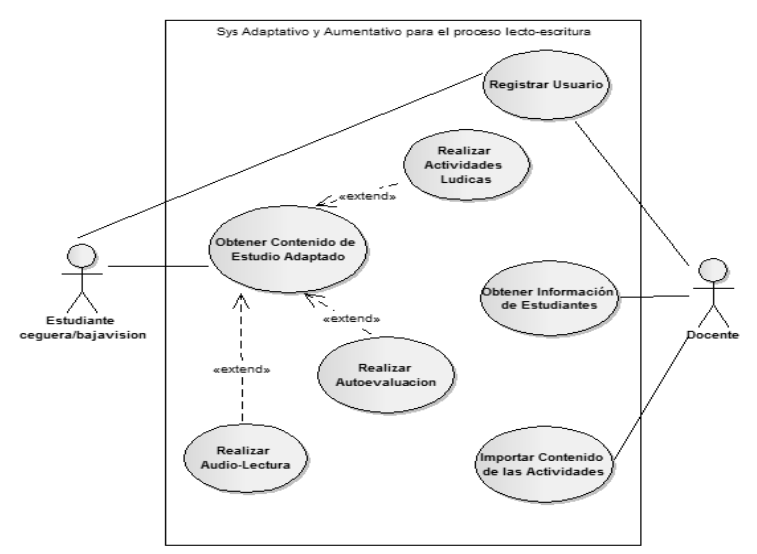

Fig. 3. Diagrama de caso de uso del sistema.

\subsection{Arquitectura del sistema}

El Sistema compuesto por los Modelos de Dominio, Adaptación y Usuario requiere obtener el perfil del usuario para lograr la interacción de los distintos dispositivos de Interfaces Naturales activos de acuerdo a la tecnología de apoyo para la discapacidad determinada (Baja visión/ceguera) y acceder a los contenidos mediante los Objetos de Aprendizaje, los cuales son considerados como recursos de aprendizaje compuesto por los cuatro elementos: Competencia, contenido de información, actividad de aprendizaje y la evaluación. Para el registro de la información y seguimiento acadé- 
mico se utilizó una base de datos en MySQL como se muestra la estructura general del sistema en la Figura 4.

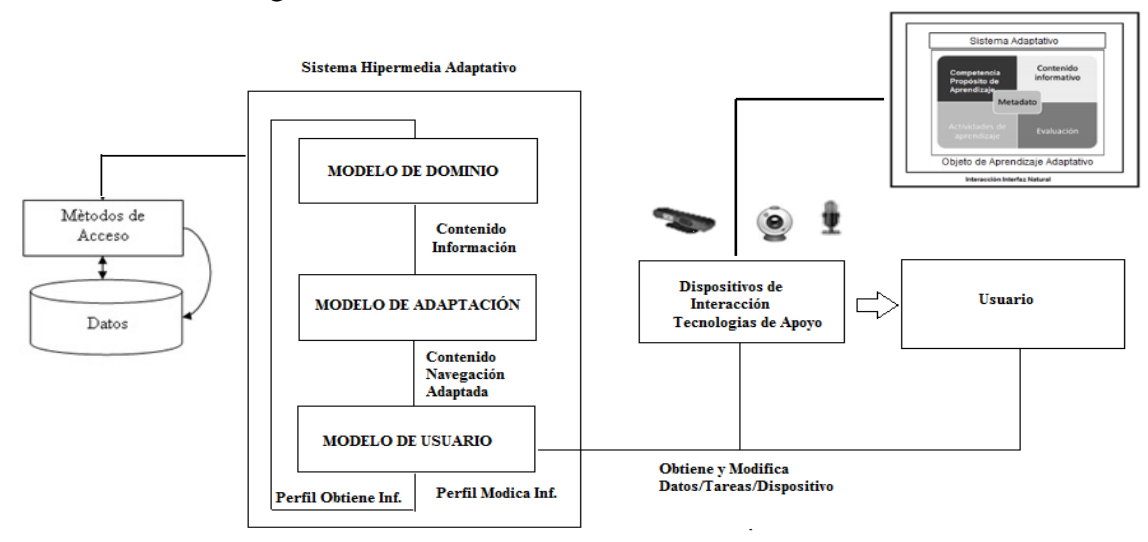

Fig. 4. Estructura general del sistema.

El sistema considera tres niveles de desempeño de las competencias son: 1) Iniciada, 2) En Proceso y 3) Desarrollada. De acuerdo al perfil pueden ser: Principiante, Intermedio y Avanzado. Para evaluar los contenidos se maneja en tres puntajes: 1) Bajo, menor a la media, 2) Regular, dentro de la media y 3) Alto, superior a la media, lo cual permite llevar el seguimiento académico de su proceso de aprendizaje.

\section{Desarrollo y pruebas del sistema}

Para desarrollo del sistema se usó Greenhouse SDK que permite la interacción de varios usuarios a través de la entrada gestual, voz y del dispositivo, manejo de múltiples pantallas, y en red, versión para Mac OS X. El sistema utiliza el reconocimiento de gestos y de voz, un gesto es el movimiento o posicionamiento resultante de la combinación de varios puntos del cuerpo como por ejemplo el movimiento de las manos o la producción de señas con los dedos que permite la manipulación de los objetos del sistema. Los gestos que se reconocen para el sistema son: A) Victory Pose (Posición victoria), B) ClosedFistPose (Posición puño cerrado), C) NumberonePose (Posicion numero uno), D) OpenPalmFingersSpreadPose (Posición de Mano abierta y dedos separados), E) LShapePose (Posición figura de L) F) OpenPalmFingersTogetherPose (Posición de Mano abierta dedos juntos). Tal como se muestra en la figura 5.

Así también se proporciona una clasificación de los eventos del usuario en tres formas principales: dejar escapar eventos, apuntar eventos y desplazamiento de eventos GreenHouse proporciona un lenguaje en común que puede ser utilizado para definir métodos o acciones que generan los diferentes dispositivos de entrada, como son el mouse, teclado, dispositivos móviles, y gestos captados por el Kinect. La conexión entre dispositivo y el sistema para su desarrollo se realizó mediante SDK de Open NI (NITE) y/o por medio del SDK de Greenhouse, los cuales permiten la creación de una 
interfaz de reconocimiento gestual y de comandos de voz. Esto permitió la construcción de archivos y/o scripts en C++ y C\# y Librería Speech y Recognition en Java correspondientes para su implementación como se muestra en la Figura 6.
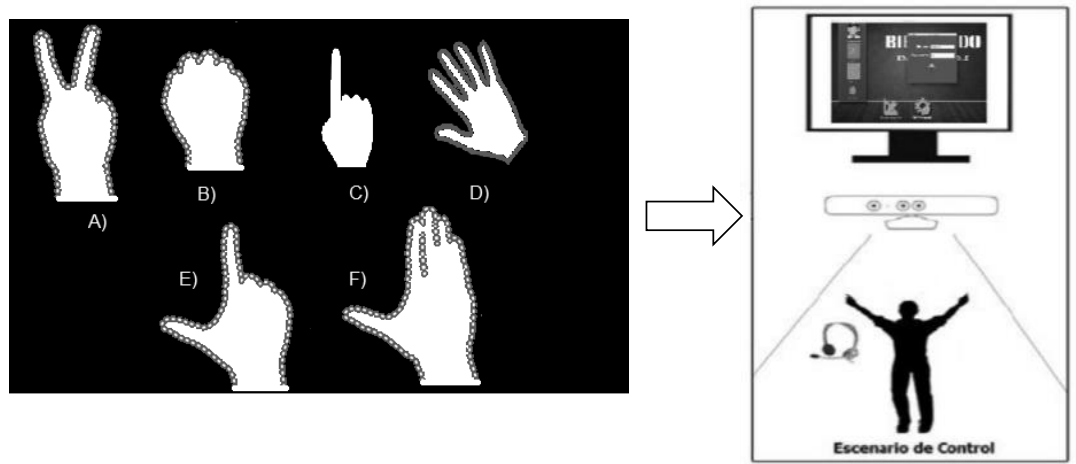

Fig. 5. Reconocimiento de gestos del sistema.

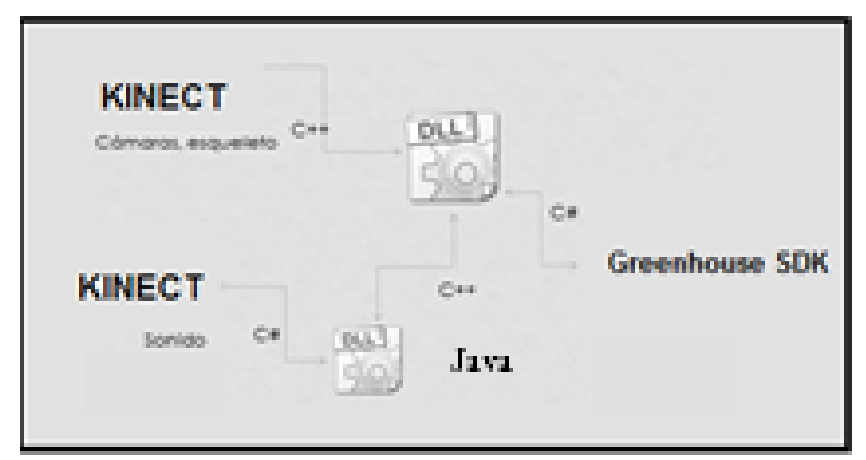

Fig. 6. Herramientas para el reconocimiento de gestos y voz del sistema.

Las personas con discapacidad visual utilizan otros sentidos para la percepción como el tacto y el oído, por lo cual el sistema permite manejar y estimular estos dos sentidos e incluso el visual dependiendo del tipo de discapacidad visual del usuario. Por lo cual el sistema se diseñó para lograr una interacción basada en gestos (movimientos o posicionamiento) y voz logrando una mayor interacción con el sistema y proporcionando una estimulación física y cognitiva para el apoyo del aprendizaje de los procesos de lectura y escritura. Como resultados de la aplicación se muestran algunas de las pantallas del sistema.

En el formulario el administrador docente podrá registrar sus datos para obtener acceso al sistema y posteriormente registrar los datos de su alumna/o para llevar un control de sus avances en las actividades y dar acceso al uso del sistema como se muestra en la Figura 7. 


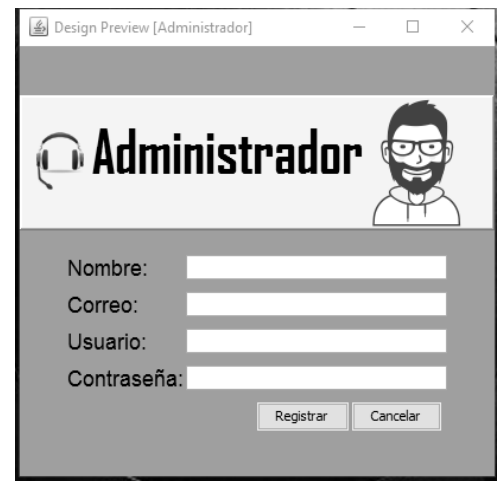

Fig. 7. Interfaz de registro administrador.

Un ejemplo de una actividad de nivel inicial después de recorrer el objeto de aprendizaje responde la evaluación de forma textual y auditivamente, cada respuesta correcta se registra y le permite ir avanzando en los contenidos de cada nivel para poder desarrollar las competencias de lectura y escritura, como se muestra en la Figura 8.
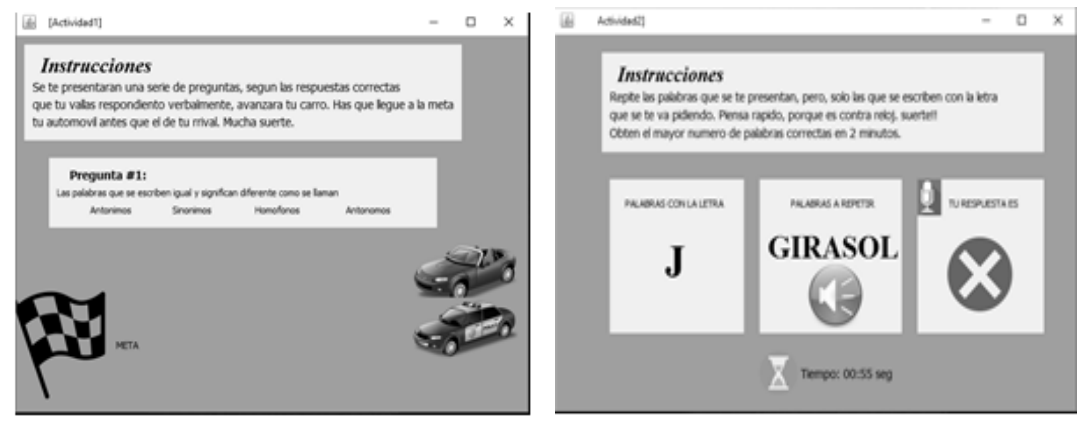

Fig. 8. Actividades de nivel 1 y nivel 2 respectivamente.

En las actividades de nivel 2 donde el usuario debe ir repitiendo acciones y palabras que se le indican, el objetivo es poder interactuar y para poder adquirir habilidad para ir acumulando puntos, ya que se irá reduciendo el tiempo de respuesta.

\subsection{Prueba piloto}

El prototipo del sistema fue piloteado en un grupo de 15 usuarios, los cuales poseen baja visión. Con respecto a las pruebas de funcionalidad se presentaron tres posibles escenarios posibles para los usuarios finales, a continuación se describen:

- Situación 1: Al usuario alumno se le dió una breve explicación del uso del sistema y se le acompaño en cada actividad. 
- Situación 2: Al usuario alumno se le explicó el uso del Sistema y no se le acompañó en las actividades.

- Situación 3: Al usuario alumno no se le explicó el uso del Sistema y solo se le acompañó al inicio de una actividad.

Para cada una de las situaciones los usuarios deberán cumplir ciertas tareas para así comprobar el funcionamiento del uso de los Objetos de Aprendizaje Adaptativos y del sistema.

- Tarea 1: Poder ser detectado por el dispositivo del Kinect y registrar su perfil (Alumno con discapacidad /sin discapacidad).

- Tarea 2: Encontrar el menú de navegación y seleccionar una opción.

- Tarea 3: Utilizar los objetos de aprendizaje y recorrerlos mediante la interacción del dispositivo del Kinect y de comandos de voz.

- Tarea 4: Realizar las autoevaluaciones y reconocer su nivel de competencia.

Después de realizar las pruebas a 15 usuarios con 5 por cada situación, se muestran los resultados obtenidos en la Tabla 1, donde se observa el porcentaje de usuarios que pudieron completar la tarea.

Tabla 1. Resultados obtenidos de la prueba piloto.

\begin{tabular}{cccc}
\hline & \multicolumn{3}{c}{ Escenarios de Test } \\
\hline Tareas & $\begin{array}{c}\text { Situación } \\
\text { No. 1 }\end{array}$ & $\begin{array}{c}\text { Situación } \\
\text { No. 2 }\end{array}$ & $\begin{array}{c}\text { Situación } \\
\text { No. 3 }\end{array}$ \\
\hline Tarea no.1 & $90 \%$ & $85 \%$ & $70 \%$ \\
Tarea no.2 & $100 \%$ & $80 \%$ & $85 \%$ \\
Tarea no.3 & $90 \%$ & $85 \%$ & $80 \%$ \\
Tarea no.4 & $90 \%$ & $80 \%$ & $90 \%$ \\
\hline Promedio & $92.5 \%$ & $82.5 \%$ & $81.2 \%$ \\
\hline
\end{tabular}

Para los usuarios con baja visión con una breve explicación de la Situación No. 1 el desempeño fue del $92.5 \%$ del cumplimiento de las tareas mientras que los usuarios de la Situación 2 al $82.5 \%$ y para la situación 3 lograron las tareas solo en un $81.2 \%$ esto implica que se presenta una interfaz intuitiva, sencilla y agradable para personas con discapacidad visual.

Finalmente se aplicó una encuesta de satisfacción "Valoración del Software [12], la cual evalúa siete criterios: Navegación, Interactividad, Inmersión, Usabilidad, Creatividad, Efectividad y Calidad, con una escala de 1 a 5 , cuyo promedio obtenido fue de $4.5(91.7 \%)$, a continuación, se presentan los resultados en la Figura 11. 


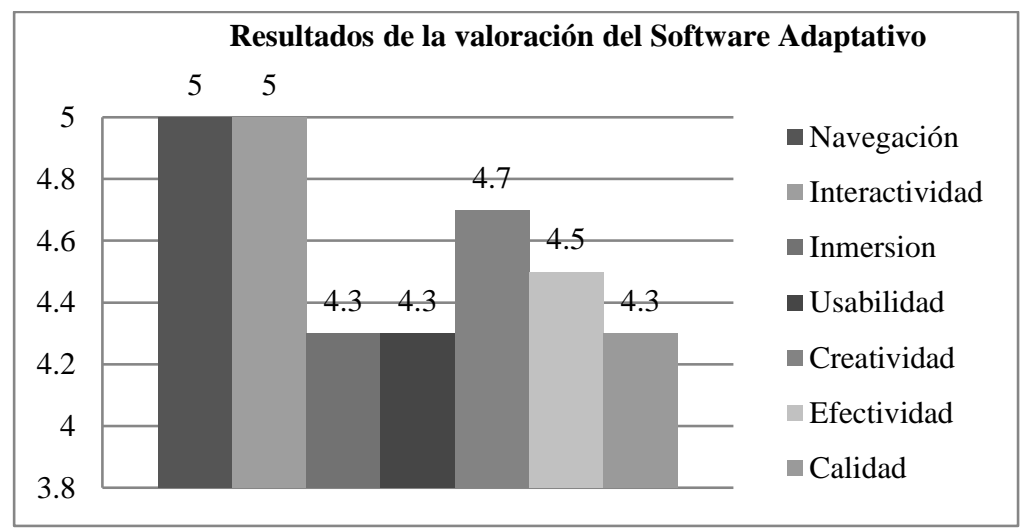

Fig. 9. Resultados de la encuesta de valoración del sistema.

\section{Conclusiones y trabajo a futuro}

Una de las principales contribuciones del Software es la adaptación a las necesidades del perfil del usuario con discapacidad y que mediante el uso interfaces naturales, el reconocimiento por medio de la voz de los contenidos de aprendizaje aportan una mayor interacción y ejecución, lo cual permite motivar a los estudiantes con discapacidad visual siendo una alternativa dinámica de aprendizaje para apoyar el proceso de adquisición de las competencias de lectura y escritura.

Como una perspectiva de este trabajo es realizar la evaluación de la accesibilidad y usabilidad del sistema propuesto ampliando la muestra de estudio y poder proveer a los alumnos con discapacidad materiales y herramientas digitales que puedan enriquecer el proceso de aprendizaje en distintas asignaturas, apoyar su vida académica y promover la inclusión del uso de las TIC para los estudiantes con alguna discapacidad para disminuir la brecha digital.

\section{Referencias}

1. Robles, M: Percepción visual y ceguera. Aspectos evolutivos y educativos de la deficiencia visual. Madrid, ONCE, http://www. once.es/new (1999)

2. Instituto Nacional de Estadística y Geografía (INEGI): Encuesta Nacional de Ingresos y Gastos de los Hogares, http://www.inegi.org.mx/est /contenidos/proyectos/encuestas/hogares/(2012)

3. Vázquez, J.: Clasificación Internacional del Funcionamiento, de la Discapacidad y de la Salud: CIF, Organización Mundial de la Salud. España http://www.imserso.es/InterPresent2/groups/imserso/documents /binario/435cif.pdf (2001)

4. Alcantud, F.: Estudiantes con Discapacidades Integrados en los Estudios Universitarios. Manual de Asesoramiento y Orientación Vocacional, Primera Edición, Síntesis, Madrid (1995) 
Desarrollo de un sistema aumentativo y adaptativo para apoyar el proceso de lectoescritura ...

5. AENOR (Asociación Española de Normalización y Certificación), productos de apoyo para personas en situación de discapacidad. Clasificación y terminología (UNE-EN ISO9999), http://www.aenor.es/aenor/normas (2017)

6. Gaudioso, Vázquez, E.: Contribuciones al Modelado del Usuario en Entornos Adaptativos de Aprendizaje y Colaboración a través de Internet mediante técnicas de aprendizaje Automático. Tesis doctoral, Facultad de Ciencias, Universidad Nacional de Educación a Distancia, Madrid, España (2002)

7. Brusilovsky, P.: Methods and techniques of adaptive hypermedia. User Modeling and User Adapted Interaction, (Special issue on adaptative hypertext and hypermedia), 6, 87-129 (1996)

8. Brusilovsky, P., Millan, P.: User Models for Adaptive Hypermedia y Adaptive Educational Systems. The Adaptiv, Lecture Notes in Computer Science, 4321, 3-53 (2007)

9. Valli, A.: Notes on natural interaction. http://www.idemployee.id. tue.nl/g.w.m.rauterberg/Movies/NotesonNatural Interaction.pdf (2015)

10. Microsoft Kinect Sensor, https://www.microsoft.com/enus/kinectforwindows (2015)

11. Leap Motion Controller. https: //www . leapmotion.com/(2015)

12. Myo Gesture Control Armband. https://www. thalmic.com/myo (2015)

13. Google Glass. https://developers.google.com/glass

14. Wigdor, D., Wixon, D.: Brave NUI World, Designing Natural User Interfaces for Touch and Gesture. http://dl.acm.org/citation. cfm?id=1995309 (2011)

15. Tobón, S., Pimienta, J., García, J.: Secuencias didácticas: Aprendizaje y Evaluación de Competencias. México, Pearson- Prentice Hall (2010) 